\title{
Hierarchical nanoporous microtubes for high-speed catalytic microengines
}

\author{
Jinxing Li ${ }^{1,2}$, Zhaoqian Liu ${ }^{1,2}$, Gaoshan Huang ${ }^{1}$, Zhenghua $\mathrm{An}^{3}$, Gang Chen ${ }^{4}$, Jing Zhang ${ }^{1,2}$, Menglin $\mathrm{Li}^{1}$, \\ Ran $\mathrm{Liu}^{2}$ and Yongfeng $\mathrm{Mei}^{1}$
}

Powerful micro-/nano-motors with high speeds and large driving forces in fluids are of great importance in propelling micro-I nanomachines for various tasks. Here, we achieved highly efficient catalytic locomotion in microtubular engines with hierarchical nanoporous walls. The sophisticated structures provide an enlarged surface area and improved reactant accessibility, which remarkably enhanced their catalytic activity toward $\mathrm{H}_{2} \mathrm{O}_{2}$ decomposition, accelerating the microengine's speed. The fast catalytic locomotion of such hierarchical nanoporous microtubes makes them excellent candidates as efficient micro-machines for biomedical applications.

NPG Asia Materials (2014) 6, e94; doi:10.1038/am.2014.11; published online 4 April 2014

Keywords: catalytic locomotion; hierarchical structure; nanoporous; tubular microengine

\section{INTRODUCTION}

Directly moving micro-/nano-objects is of great importance in numerous potential applications, such as intelligent drug-delivery, smart nanomachinery and nanoscale assembly, ${ }^{1-3}$ which require the design and fabrication of powerful micro-/nanoengines to overcome the Brownian motion and viscous forces that become significant at such small size scales. ${ }^{4}$ Such engines, which have been produced by nature through millions of years of evolution, have the ability to convert chemical energy to mechanical energy. For instance, kinesin can induce the locomotion of microtubules by deriving energy from adenosine triphosphate molecules. ${ }^{5}$ Man-made devices mimicking their natural counterparts have been achieved in recent years. ${ }^{6,7}$ Although these devices are advantageous in autonomous movement, the inelasticity of biomaterials in in vitro environments leads to limited lifetimes. ${ }^{8}$ Nevertheless, the goal of developing self-regulating engines has inspired investigations into artificial micro-/nanoengines that operate on locally supplied chemical fuels (in contrast to biomaterials). ${ }^{8}$ Typical structures in the form of wires, ${ }^{9-11}$ spheres ${ }^{12,13}$ or tubes ${ }^{14-17}$ have been demonstrated to accomplish controlled movement in chemical fuels by utilizing suitable catalysts and structural asymmetry. ${ }^{2}$ In most cases, energy is converted based on the chemical catalytic decomposition of $\mathrm{H}_{2} \mathrm{O}_{2}$ to power the movement of the engines. Preliminary demonstrations of multi-functional engines performing complex tasks, such as dynamic target transportation and isolation under remote control using an external field, have been reported so far. ${ }^{18-20}$
The mobility of catalytic micro-/nanomotors was found to be intrinsically determined by the kinetics of the catalytic reaction and intimately related to factors such as the temperature and electrical potential, the concentration of the chemical fuel and the geometry of the motors. ${ }^{21,22}$ Various approaches have been employed to boost the catalytic reaction rate and in turn increase the motor's speed. ${ }^{23,24}$ Tailoring the motors' geometry is considered to be an especially effective method. For instance, the incorporation of carbon nanotubes within the Pt segments, ${ }^{25}$ enhancing the surface roughness of $\mathrm{Pt}^{26}$ and the assembly of $\mathrm{Pt}$ nanoparticles in microtubes ${ }^{27}$ have been used to enhance the speeds of self-propelled motors. Despite these remarkable progresses in improving the performance of catalytic motors, their energy-conversion efficiency is significantly lower than those found in natural biosystems. As a result, the enhancement of their energyconversion efficiency remains a crucial objective.

One promising approach is to improve the electrochemical activity inside the microtubular reactor by modifying its surface morphology and incorporating specially designed hierarchical architectures. Herein, we report for the first time the preparation of a novel tubular microengine consisting of a hollow catalytic reactor with hierarchical nanoporous walls based on strain-engineered rolled-up nanotechnology by using anodic aluminum oxide (AAO) as a sacrificial template. Such nanoporous catalytic tubular microengines with open porosity in all dimensions beneficially allow the unlimited transport of the molecules in the fuel medium. In addition, the nanoporous microengines possess a higher order geometric complexity and a higher surface area to volume ratio compared with smooth microengines.

${ }^{1}$ Department of Materials Science, Fudan University, Shanghai, People's Republic of China; ${ }^{2}$ State Key Lab of ASIC and System, Fudan University, Shanghai, People's Republic of China; Institute of Advanced Materials and State Key Laboratory of Surface Physics, Fudan University, Shanghai, People's Republic of China and ${ }^{4}$ School of Pharmacy, Fudan University, Shanghai, People's Republic of China

Correspondence: Professor G Huang or Professor Y Mei, Department of Materials Science, Fudan University, 220 Handan Road, Shanghai 200433, People's Republic of China. E-mail: gshuang@fudan.edu.cn or yongfeng.mei@gmail.com

Received 12 April 2013; revised 4 December 2013; accepted 8 December 2013 
Nanoporous microengines with different nanopore size distributions fueled by hydrogen peroxide were investigated, and a 2-5-fold enhancement in microengine speed relative to smooth microengines was statistically observed. Furthermore, we demonstrated that a hydrogen peroxide fuel solution with a concentration of only $0.2 \%$ is sufficient to propel the nanoporous microengine at a speed of $120 \mu \mathrm{m} \mathrm{s}^{-1}$, which greatly extends the feasibility of catalytic microengines for biomedical applications. As such, the novel nanoporous microengines can provide new possibilities for designing and producing ultra-powerful micro-/nanomachines. Moreover, the hierarchical nanoporous Pt microtubes show fast mass transfer and superior electrocatalytic activity and are expected to find applications in micro-/nanofluidic systems as well as in high surface area catalysis for energy applications.

\section{EXPERIMENTAL PROCEDURES}

The fabrication of the AAO templates, the procedure for the smooth microengine preparation, the morphologies of the porous metallic nanomembranes and detailed calculations can be found in the Supplementary Information.

\section{Fabrication of nanoporous microtubular structures}

As schematically illustrated in Figure 1a (i-iii), a hybrid microtubular structure with a nanoporous wall was produced via a rolled-up process using AAO membranes as a sacrificial template (i in Figure 1a). Pre-strained $\mathrm{Ti} / \mathrm{Cr} / \mathrm{Pt}$ metallic tri-layers with thicknesses of $7 / 7 / 6 \mathrm{~nm}$ were subsequently deposited on the AAO membranes by e-beam evaporation at $2 \times 10^{-4} \mathrm{~Pa}$ (ii in Figure 1a). $\mathrm{Ti} / \mathrm{Cr}$ nanomembrane was adopted to build a strain gradient in the nanomembrane, facilitating the subsequent rolling-up process. Pt was used as a catalytic layer for $\mathrm{H}_{2} \mathrm{O}_{2}$ decomposition. The metal deposition rate used was as low as $0.1 \AA \mathrm{s}^{-1}$ to avoid blocking the nanopore openings. The top surface morphology of the deposited metallic tri-layers precisely mirrored the AAO membranes below but with a smaller pore size due to the coating effect, as demonstrated in Supplementary Figure S1. After metal deposition, a fine mechanical scratching process was employed to divide the upper metallic tri-layers into quadrate patterns with a size of $10-30 \mu \mathrm{m}$. Finally, AAO sacrificial templates were selectively removed by a $20 \% \mathrm{KOH}$ solution, releasing the patterned metallic tri-layers. The intrinsic stress in the Ti/Cr/Pt tri-layers made them automatically roll into microtubular structures (iii in Figure 1a), which were then dried in a critical point dryer (Leica CPD 030) using liquid $\mathrm{CO}_{2}$ as the intermediary to avoid collapse caused by the surface tension of the aqueous etchants.

\section{Optical microscopy of catalytic motion}

The motions of the microengines were captured by a Keyence VHX-60 optical microscope (up to 50 frames $^{-1}$ ), and the trajectories were analyzed in detail with the assistance of a particle-tracking plug-in for Image J.

\section{RESULTS AND DISCUSSION}

The fabrication strategy was based on rolled-up nanotechnology $y^{28,29}$ using AAO membranes ${ }^{30,31}$ as a sacrificial template, as schematically illustrated in Figure 1a (i-iii). Figure 1b shows a scanning electron microscopy image of a nanoporous microtube (that is, microtubular engine) rolled up from porous $\mathrm{Ti} / \mathrm{Cr} / \mathrm{Pt}(7 / 7 / 6 \mathrm{~nm})$ nanomembranes using a sacrificial AAO template with small pore diameters of $\sim 150 \mathrm{~nm}$. Interestingly, the metallic layers that were deposited into the nanochannels of the AAO membrane by the e-beam evaporation procedure formed metallic nanotubes (ii in Figure 1a). During the following rolling process, the metallic nanotubes formed in the nanochannels of the AAO membrane were also rolled up with the metallic tri-layer, resulting in nanotubes similar to cotton fibers and with a uniform outer diameter being presented on the outer surface of the microtube, forming a hierarchical nanoporous structure. In addition, because only a limited amount of metal penetrated into the nanochannels, the walls of the nanotubes were very thin, making the surface look hairy.

Similar to typical rolled-up microstructures, the geometries of the nanoporous microtubes could be controlled by varying the shape and thickness of the metallic nanomembrane. In the present work, the
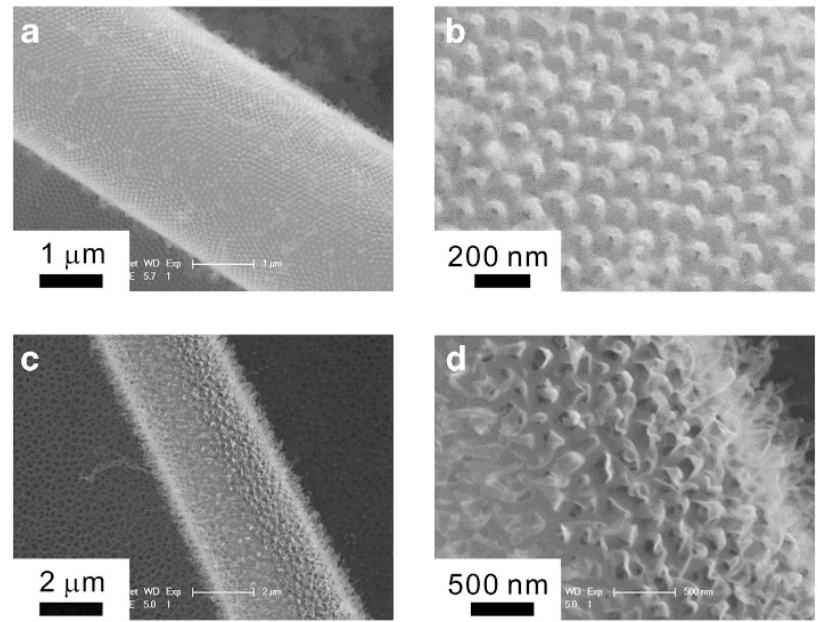

Figure 2 (a) Scanning electron microscopy (SEM) image of a nanoporous microtube with small pore diameters $(d \approx 20 \mathrm{~nm})$ and $(\mathbf{b})$ a close-up of the corresponding surface morphology. (c) SEM image of a nanoporous microtube with large pore diameters $(d \approx 150 \mathrm{~nm})$ and $(\mathbf{d})$ a close-up of the corresponding surface morphology.
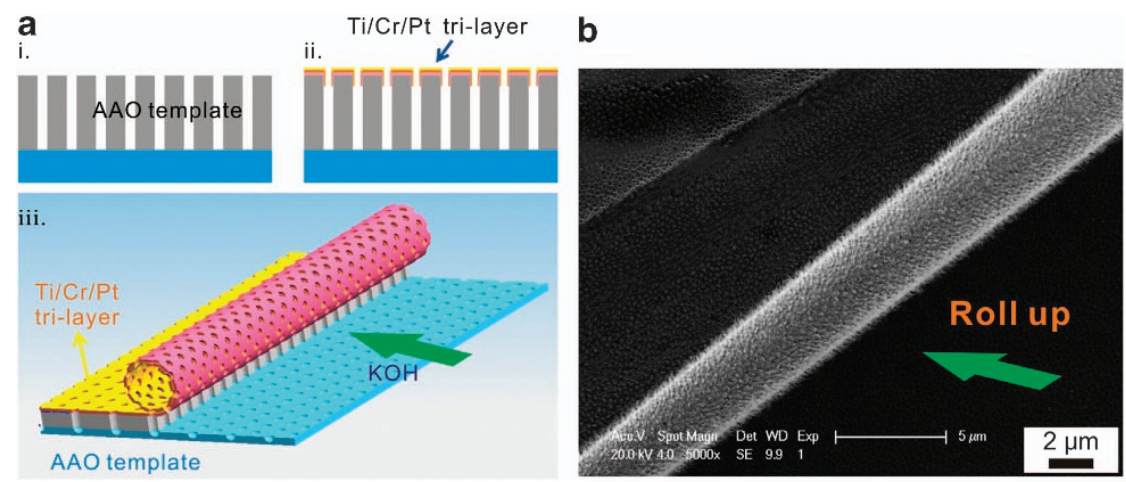

Figure 1 (a) Three-dimensional schematic illustration of the fabrication method for the nanoporous microtubes. (b) A typical scanning electron microscopy (SEM) image of a nanoporous microtube (that is, microtubular engine) rolled up from porous Ti/Cr/Pt (7/7/6 nm) nanomembranes using a sacrificial AAO template with small pore diameters of $\sim 150 \mathrm{~nm}$. 
diameter of the microtubes was $\sim 4-10 \mu \mathrm{m}$, and the length ranged from $10-40 \mu \mathrm{m}$. To characterize the nanoporous microengines' catalytic propulsion performance, two different nanopore size distributions with large $(d \approx 150 \mathrm{~nm})$ and small pores $(d \approx 20 \mathrm{~nm})$ were prepared and studied. We also fabricated rolled-up microtubes with smooth tube walls from $\mathrm{Ti} / \mathrm{Cr} / \mathrm{Pt}$ nanomembranes for comparison, and the details of the procedure can be found in the Supplementary Information. Figure $2 \mathrm{a}$ shows a nanoporous microtube with small pore diameters of $\sim 20 \mathrm{~nm}$. The corresponding enlarged surface morphology is displayed in Figure 2b, where an ordered nanopore array with a pore density of $\sim 10^{14} \mathrm{~m}^{-2}$ can be clearly observed (see also Supplementary Figure S1). Compared with conventional smooth microtubes of the same diameter and length, the inner Pt surface area of the nanoporous microtubes was approximately twice as large due to the high density of nanotubes (Supplementary Information). Figures $2 \mathrm{c}$ and $\mathrm{d}$ show a nanoporous microtube with a large pore size of $\sim 150 \mathrm{~nm}$. In this case, the evaporated metal atoms and clusters can penetrate much farther into the nanochannels of the AAO membrane during the deposition process, and the length of the metallic nanotubes therefore increased to $\sim 500 \mathrm{~nm}$ (Figures $2 \mathrm{c}$ and $\mathrm{d}$ ). The inner surface area was at least nine times larger than that of the smooth microtube (Supplementary Information).

The catalytic motions of these nanoporous microtubular engines (microtubes) were monitored in a $5 \% \mathrm{H}_{2} \mathrm{O}_{2}$ solution. The $\mathrm{H}_{2} \mathrm{O}_{2}$ was

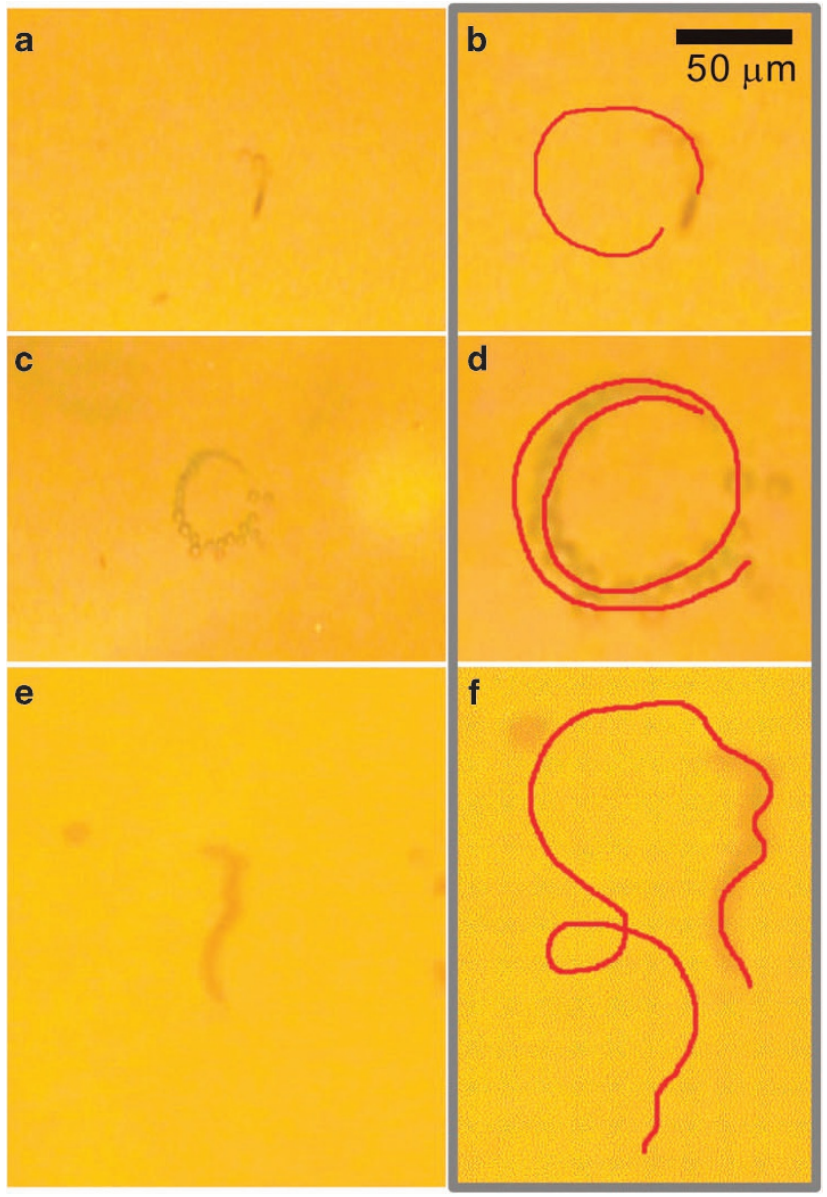

Figure 3 Selected motion images (a, c, e) and tracking trajectories (b, d, f) of microengines over a time period of $1 \mathrm{~s}$ in $5 \% \mathrm{H}_{2} \mathrm{O}_{2}$ : (a, b) smooth microengine, (c, d) nanoporous microengine with small pores, and (e, f) nanoporous microengine with large pores. catalytically decomposed by the inner Pt surface, causing gaseous $\mathrm{O}_{2}$ microbubbles to form inside the microtubular channel (the main channel). The shape of the rolled-up structures was slightly asymmetrical, ${ }^{14}$ causing the $\mathrm{O}_{2}$ microbubbles in the main channel to move toward the larger opening due to the pressure difference, where they were then expelled, propelling the engine in the opposite direction. ${ }^{14,32,33}$ Figure 3 illustrates the locomotion of microengines with identical diameters $(4 \mu \mathrm{m})$ and lengths $(20 \mu \mathrm{m})$ but different nanopore diameters. Their tracking trajectories and moving distances over $1 \mathrm{~s}$ are presented in the right column. The movement of a conventional smooth microengine is also presented for comparison. One may note that the microengines swim in circular paths, which is mainly due to the asymmetry in the microtubular structure. The asymmetry in the structure makes the force perpendicular to the tube axis unbalanced and thus rotates the microengine during selfpropulsion. In addition, as demonstrated in the Supplementary Videos $1-3$, the smooth microengine moves $>180 \mu \mathrm{m}$ with an $\mathrm{O}_{2}$ microbubble generation frequency of $\sim 20 \mathrm{~Hz}$, whereas the microengine with small nanopores $(\sim 20 \mathrm{~nm})$ moves $>550 \mu \mathrm{m}$ with an $\mathrm{O}_{2}$ microbubble generation frequency of $\sim 60 \mathrm{~Hz}$, and the microengine with large nanopores $(\sim 150 \mathrm{~nm})$ moves even faster. These results
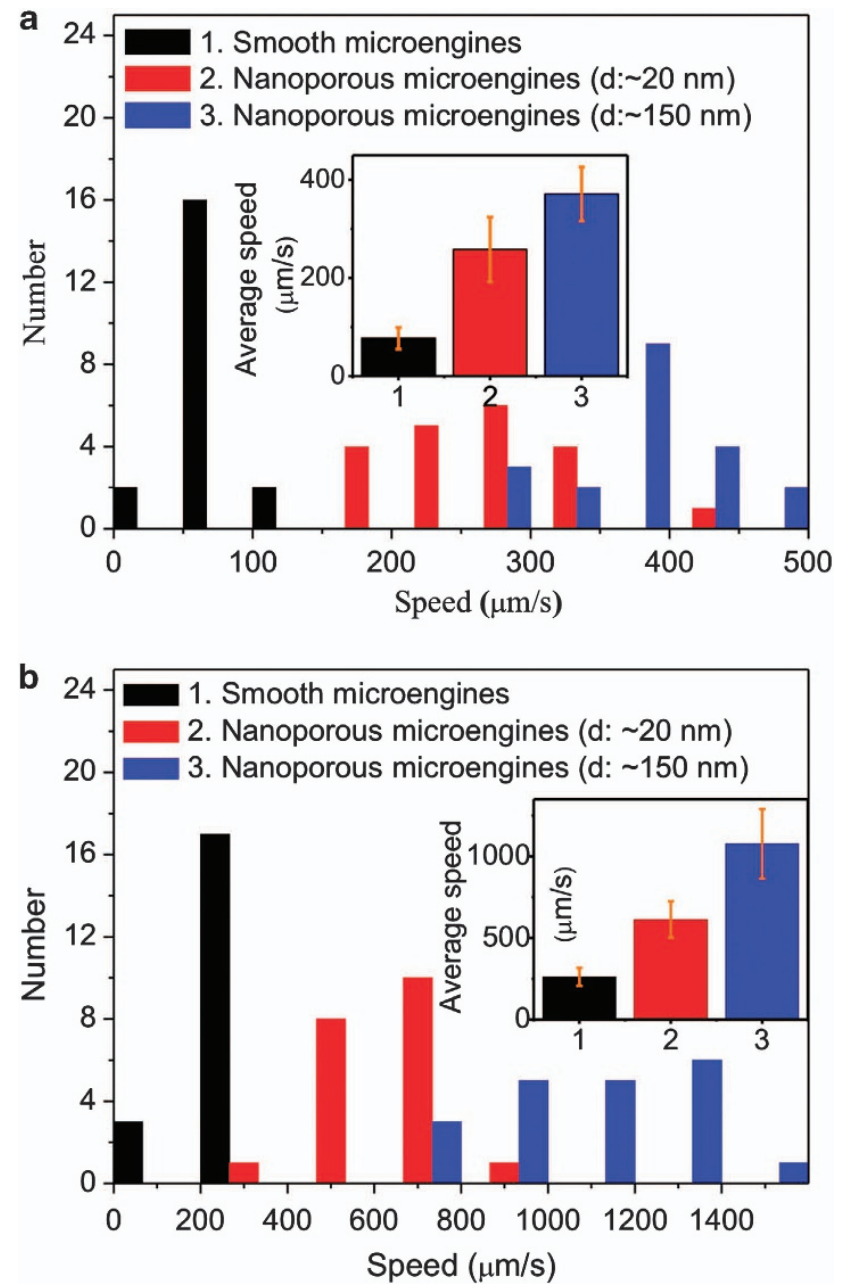

Figure 4 Acceleration of nanoporous microengines. The histograms indicate the speed distribution of the three types of microengines in (a) a $3 \% \mathrm{H}_{2} \mathrm{O}_{2}$ fuel and (b) a $7 \% \mathrm{H}_{2} \mathrm{O}_{2}$ fuel. The inset bar graphs demonstrate the corresponding average speeds of the microengines with the three different geometries. 


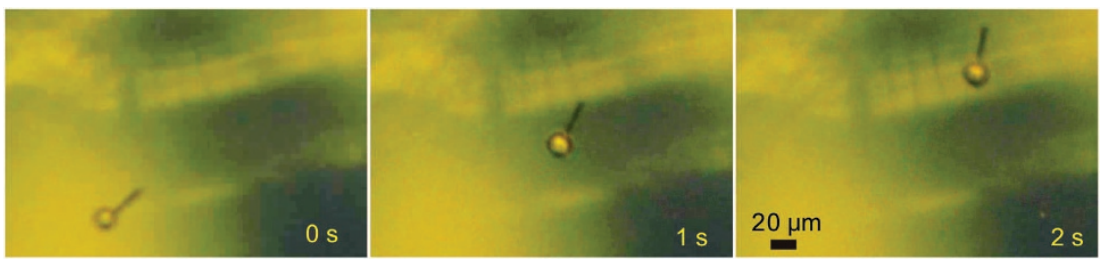

Figure 5 Time-lapse images of a moving nanoporous microengine (with 150-nm nanopores on the wall) at a low fuel concentration of $0.2 \%$.

suggest that the presence of nanopores in the walls of the engines may effectively increase their speed. To further investigate the catalytic motion behavior of the microengines, statistics for the motion speed were collected based on the experimental observations of 20 microengines with similar dimensions in each group. Figures $4 \mathrm{a}$ and $\mathrm{b}$ show the histograms of the speed distribution of selected microengines moving in $3 \%$ and $7 \% \mathrm{H}_{2} \mathrm{O}_{2}$, respectively; the average speeds are also shown in the corresponding insets. In both cases, the narrow speed distribution in the histograms shows the high consistency of the microengines' locomotion behaviors. In the $3 \% \mathrm{H}_{2} \mathrm{O}_{2}$ solution, the average speed of the nanoporous microengines with pore diameters of $20 \mathrm{~nm}$ increased from $77 \mu \mathrm{m} \mathrm{s}^{-1}$ in the case of smooth microengines to $260 \mu \mathrm{ms}^{-1}$. The highest speed of $370 \mu \mathrm{ms}^{-1}$ was achieved by nanoporous microengines with pore diameters of $150 \mathrm{~nm}$, marking a nearly fivefold enhancement in speed relative to the smooth microengines. For the $7 \% \mathrm{H}_{2} \mathrm{O}_{2}$ solution, higher average speeds of 262 , 613 and $1077 \mu \mathrm{m} \mathrm{s}^{-1}$ were obtained for the smooth microengines, nanoporous microengines with $\sim 20 \mathrm{~nm}$ pores and nanoporous microengines with $\sim 150 \mathrm{~nm}$ pores (see the Supplementary Video $4)$, respectively, as displayed in Figure $4 \mathrm{~b}$.

The mechanical power output of the microengine is the product of its speed and the drag force (Stokes' Force) it must overcome. According to the literature, the drag force is considered to be proportional to the speed, ${ }^{21,34}$ and, therefore, the output power is proportional to the square of the speed. From our results, we see that the power of a microengine with large nanopores is $>10$ times higher than that of a smooth microengine. In addition, we note that the increased speeds of the nanoporous microengines with respect to the smooth microengine are associated with enhanced $\mathrm{O}_{2}$ microbubble generation frequency. This effect is believed to be partially due to the accelerated catalytic decomposition from the hierarchical wall and a corresponding increase in the catalytic surface area, which leads to a faster $\mathrm{O}_{2}$ production rate. In fact, we hypothesize that the accelerated reaction is due not only to the increased surface but also to the fast reactant transfer rate inside the microtubular channel. For microtubular engines, the microchannel serves both as the chemical reaction chamber and as the oxygen-collecting cavity, where $\mathrm{H}_{2} \mathrm{O}_{2}$ is pumped into the tubular reactor from the front end and bubbles are expelled from the back end. ${ }^{14,18}$ This liquid flow feeds the catalytic reaction, leading to the continuous motion of the microengine. ${ }^{14}$ For smooth microengines, the chemical fuel can only be pumped in from the front opening, and the reaction may actually be limited by an $\mathrm{H}_{2} \mathrm{O}_{2}$ shortage, especially during fast decomposition (Supplementary Figure S2). This is unlikely to happen in microengines with nanoporous walls because the nanopores in the tube wall can serve as transport channels for $\mathrm{H}_{2} \mathrm{O}_{2}$ (Supplementary Figure S2). The increased mass transport rate results in fast filling of the reactor with fresh $\mathrm{H}_{2} \mathrm{O}_{2}$, supporting even faster decomposition. The nanoporous microengine with large pores enables a faster transportation rate and a higher reaction rate compared with the smooth microengines and nanoporous microengines with small pores. The high production rate correspondingly causes fast accumulation of $\mathrm{O}_{2}$ and the formation of microbubbles, ${ }^{18}$ which are then expelled from the back end of the microtubular channel, generating a recoiling force. A quantitative analysis of this motion is challenging at the present stage, but our experimental results undoubtedly prove that the nanoporous structures on the microtube walls enhance the reaction rate and motion speed by increasing the reactant transfer rate and the surface area.

Moreover, the increase in the reaction rate also leads to a decrease in the $\mathrm{H}_{2} \mathrm{O}_{2}$ concentration threshold for switching on the engine. In our experiments, we found that the threshold can be as low as $0.2 \%$ (compared with $1 \%$ for smooth microengines). The time-lapse images in Figure 5 display a nanoporous microengine with $150-\mathrm{nm}$ nanopores on the wall of the tubular structure moving in a $0.2 \%$ $\mathrm{H}_{2} \mathrm{O}_{2}$ solution. Although the oxygen bubble generation frequency is low, the microengine is nonetheless self-propelled at a speed of $\sim 120 \mu \mathrm{m} \mathrm{s}^{-1}$ (Supplementary Video 5). As high concentrations of hydrogen peroxide are harmful to biomaterials and tissues, ${ }^{35}$ our high-speed nanoporous microengines are highly favorable for biomaterial transportation and bioprocess visualization using chemically powered micro-/nanomachines.

\section{CONCLUSIONS}

The ideal combination of rolled-up nanotechnology and nanoporous templates enabled the fabrication of microtubular engines with hierarchical walls. Compared with normal smooth tubular microengines, the increased catalytic activity of the novel nanoporous catalytic reactor as well as its improved reactant accessibility and larger surface area due to the presence of nanopores/nanochannels in the wall of the tubular reactor enabled a dramatic acceleration of the catalytic reaction. The high-speed and low-threshold propulsion of the nanoporous microengines provides new possibilities for producing ultra-powerful micro-/nanomotors and machines with potential applications in catalysis support, ionic batteries, drug/gene delivery and medical imaging/diagnostics.

\section{CONFLICT OF INTEREST}

The authors declare no conflict of interest.

\section{ACKNOWLEDGEMENTS}

This work was supported by the Natural Science Foundation of China (Nos 51322201 and 51102049), the 'Shu Guang' project by the Shanghai Municipal Education Commission and the Shanghai Education Development Foundation, the National Basic Research Program of China (Grant No. 2011BAF06B01), a Project Based Personnel Exchange Program with CSC and DAAD, a Specialized Research Fund for the Doctoral Program of Higher Education (No. 20120071110025) and the Science and Technology Commission of Shanghai Municipality (No. 12520706300).

Author contributions: YM, JL, GH and RL designed and conceived the experiment. JL, ZL, GC, JZ, ZA and ML performed the experiments. YM, GH and JL analyzed the data, discussed it with all authors and wrote the paper. $\mathrm{JL}$ and ZL contribute this work equally. 
1 Wang, J. Nanomachines: Fundamentals and Applications (Wiley-VCH, Weinheim, Germany, 2013).

2 Paxton, W. F., Sundararajan, S., Mallouk, T. E. \& Sen, A. Chemical locomotion. Angew. Chem. Int. Ed. 45, 5420-5429 (2006).

$3 \mathrm{He}$, Y. P., Wu, J. S. \& Zhao, Y. P. Designing catalytic nanomotors by dynamic shadowing growth. Nano Lett. 5, 1369-1375 (2007).

4 Purcell, E. M. Life at low Reynolds number. Am. J. Phys. 45, 3-11 (1977),

5 Vale, R. D. \& Milligan, R. A. The way things move: looking under the hood of molecular motor proteins. Science 288, 88-95 (2000)

6 Bath, A. \& Turberfield, A. J. DNA nanomachines. Nat. Nanotechnol. 2, 275-284 (2007).

7 Browne, W. R. \& Feringa, B. L. Making molecular machines work. Nat. Nanotechnol. 1, 25-35 (2006).

8 Wang, J. Can man-made nanomachines compete with nature biomotors? ACS Nano 3 4-9 (2009)

9 Mirkovic, T., Zacharia, N. S., Scholes, G. D. \& Ozin, G. A. Nanolocomotion-catalytic nanomotors and nanorotors. Small 6, 159-167 (2010).

10 Wu, J., Balasubramanian, S., Kagan, D., Manesh, K. M., Campuzano, S. \& Wang, J. Motion-based DNA detection using catalytic nanomotors. Nat. Commun. 1, 36 (2010).

11 Mallouk, T. E. \& Sen, A. Powering nanorobots. Sci. Am. 300, 72-77 (2009).

12 Gibbs, J. G. \& Zhao, Y.-P. Autonomously motile catalytic nanomotors by bubble propulsion. Appl. Phys. Lett. 94, 163104 (2009).

13 Valadares, L. F., Tao, Y. G., Zacharia, N. S., Kitaev, V., Galembeck, F., Kapral, R. \& Ozin, G. A. Catalytic nanomotors: self-propelled sphere dimmers. Small 6, 565-572 (2010).

14 Solovev, A. A., Mei, Y. F., Bermúdez Ureña, E., Huang, G. S. \& Schmidt, O. G. Catalytic microtubular jet engines self-propelled by accumulated gas bubbles. Small $\mathbf{5}$, 1688-1692 (2009)

15 Mei, Y. F., Solovev, A. A., Sanchez, S. \& Schmidt, O. G. Rolled-up nanotech on polymers: from basic perception to self-propelled catalytic microengines. Chem. Soc. Rev. 40, 2109-2119 (2011).

16 Gao, W., Sattayasamitsathit, S., Orozco, J. \& Wang, J. Highly efficient catalytic microengines: template electrosynthesis of polyaniline/platinum microtubes. J. Am. Chem. Soc. 133, 11862-11864 (2011).

17 Huang, G. S., Wang, J. \& Mei, Y. F. Material considerations and locomotive capability in catalytic tubular microengines. J. Mater. Chem. 22, 6519-6525 (2012).

18 Solovev, A. A., Sanchez, S., Pumera, M., Mei, Y. F. \& Schmidt, O. G. Magnetic control of tubular catalytic microbots for the transport, assembly, and delivery of micro-objects. Adv. Funct. Mater. 20, 2430-2435 (2010).

19 Campuzano, S., Orozco, J., Kagan, D., Guix, M., Gao, W., Sattayasamitsathit, S. Claussen, J. C., Merkoçi, A. \& Wang, J. Bacterial isolation by lectin-modified microengines. Nano Lett. 12, 396-401 (2012).

20 Kagan, D., Laocharoensuk, R., Zimmerman, M., Clawson, C., Balasubramanian, S., Kang, D., Bishop, D., Sattayasamitsathit, S., Zhang, L. F. \& Wang, J. Rapid delivery of drug carriers propelled and navigated by catalytic nanoshuttles. Small 6, 2741-2747 (2010).

21 Li, J. X., Huang, G. S., Ye, M. M., Li, M. L., Liu, R. \& Mei, Y. F. Dynamics of catalytic tubular microjet engines: dependence on geometry and chemical environment. Nanoscale 3, 5083-5089 (2011).

22 Manjare, M., Yang, B. \& Zhao, Y. Bubble propelled microjets: model and experiment. J. Phys. Chem. C 117, 4657-4665 (2013).

23 Gao, W., Sattayasamitsathit, S. \& Wang, J. Catalytically propelled micro-/nanomotors: how fast can they move? Chem. Rec. 12, 224-231 (2012).

24 Liu, Z. Q., Li, J. X., Wang, J., Huang, G. S., Liu, R. \& Mei, Y. F. Small-scale heat detection using catalytic microengines irradiated by laser. Nanoscale 5, 1345-1352 (2013).

25 Laocharoensuk, R., Burdick, J. \& Wang, J. CNT-induced acceleration of catalytic nanomotors. ACS Nano 2, 1069-1075 (2008).

26 Zacharia, N. S., Sadeq, Z. S. \& Ozin, G. A. Enhanced speed of bimetallic nanorod motors by surface roughening. Chem. Commun. 5856-5858 (2009).

27 Li, J. X., Zhang, J., Gao, W., Huang, G. S., Di, Z., Liu, R., Wang, J. \& Mei, Y. F. Dry-released nanotubes and nanoengines by particle-assisted rolling. Adv. Mater. 25, 3715-3721 (2013).

28 Mei, Y. F., Huang, G. S., Solovev, A. A., Bermúdez Ureña, E., Mönch, I., Ding, F., Reindl, T., Fu, R. K. Y., Chu, P. K. \& Schmidt, O. G. Versatile approach for integrative and functionalized tubes by strain engineering of nanomembranes on polymers. Adv. Mater. 20, 4085-4090 (2008).

29 Schmidt, O. G. \& Eberl, K. Nanotechnology: thin solid films roll up into nanotubes. Nature 410, 168 (2001).

30 Masuda, H. \& Fukuda, K. Ordered metal nanohole arrays made by a two-step replication of honeycomb structures of anodic alumina. Science 268, 1466-1468 (1995).

31 Huang, G. S., Wu, X. L., Mei, Y. F., Shao, X. F. \& Siu, G. G. Strong blue emission from anodic alumina membranes with ordered nanopore array. J. Appl. Phys. 93, 582-585 (2003).

32 Eijkel, J. C. T. \& Berg van den, A. Water in micro- and nanofluidics sytems described using the water potential. Lab. Chip 5, 1202-1209 (2005).

33 Bico, J. \& Quéré, D. Self-propelling slugs. J. Fluid Mech. 467, 101-127 (2002).

34 Happle, J. \& Brenner, H. Low Reynolds Number Hydrodynamics (Prentice Hall, Englewood Cliffs, NJ, USA, 1965).

35 Winterbourn, C. C. Toxicity of iron and hydrogen peroxide: the Fenton reaction. Toxicol. Lett. 82-83, 969 (1995).

(c) This work is licensed under a Creative Commons
Attribution-NonCommercial-ShareAlike 3.0 Unported
License. To view a copy of this license, visit http://creativecommons. org/licenses/by-nc-sa/3.0/

Supplementary Information accompanies the paper on the NPG Asia Materials website (http://www.nature.com/am) 